\title{
Reaction Time Is Negatively Associated with Corpus Callosum Area in the Early Stages of CADASIL
}

\author{
(D) S. Delorme, (D)F. De Guio, (DS. Reyes, (D) A. Jabouley, (DH. Chabriat, and $\mathbb{D}_{\text {E. Jouvent }}$
} O- $\equiv$

\begin{abstract}
BACKGROUND AND PURPOSE: Reaction time was recently recognized as a marker of subtle cognitive and behavioral alterations in the early clinical stages of CADASIL, a monogenic cerebral small-vessel disease. In unselected patients with CADASIL, brain atrophy and lacunes are the main imaging correlates of disease severity, but MR imaging correlates of reaction time in mildly affected patients are unknown. We hypothesized that reaction time is independently associated with the corpus callosum area in the early clinical stages of CADASIL.
\end{abstract}

MATERIALS AND METHODS: Twenty-six patients with CADASIL without dementia (Mini-Mental State Examination score $>24$ and no cognitive symptoms) and without disability (modified Rankin Scale score $\leq 1$ ) were compared with 29 age- and sex-matched controls. Corpus callosum area was determined on 3D-T1 MR imaging sequences with validated methodology. Between-group comparisons were performed with $t$ tests or $\chi^{2}$ tests when appropriate. Relationships between reaction time and corpus callosum area were tested using linear regression modeling.

RESULTS: Reaction time was significantly related to corpus callosum area in patients (estimate $=-7.4 \times 10^{3}$, standard error $=3.3 \times 10^{3}$, $P=.03$ ) even after adjustment for age, sex, level of education, and scores of depression and apathy (estimate $=-12.2 \times 10^{3}$, standard error $\left.=3.8 \times 10^{3}, P=.005\right)$. No significant relationship was observed in controls.

CONCLUSIONS: Corpus callosum area, a simple and robust imaging parameter, appears to be an independent correlate of reaction time at the early clinical stages of CADASIL. Further studies will determine whether corpus callosum area can be used as an outcome in future clinical trials in CADASIL or in more prevalent small-vessel diseases.

ABBREVIATIONS: $\mathrm{BPF}=$ brain parenchymal fraction; $\mathrm{CC}_{\mathrm{A}}=$ corpus callosum area; $\mathrm{MB}=$ microbleeds; $\mathrm{RT}=$ reaction time; $\mathrm{SE}=$ standard error; $\mathrm{SVD}=\mathrm{small}-\mathrm{vessel}$ disease

C

cerebral autosomal dominant arteriopathy with subcortical infarcts and leukoencephalopathy (CADASIL) is a monogenic cerebral small-vessel disease (SVD) caused by mutations of the NOTCH3 gene. ${ }^{1}$ In unselected patients, brain atrophy and the volume of lacunes are the main MR imaging markers of disease severity. ${ }^{2,3}$ By contrast, in nondisabled patients without dementia, CADASIL may present without brain atrophy and with few if any lacunes, and MR imaging correlates of disease severity are

Received March 1, 2017; accepted after revision June 23.

From the University Paris Diderot (S.D., F.D.G., H.C., E.J.), Sorbonne Paris Cité, UMR-S 1161 INSERM, Paris, France; DHU NeuroVasc Sorbonne Paris Cité (F.D.G., H.C., E.J.), Paris, France; and Department of Neurology (S.R., A.J., H.C., E.J.), AP-HP, Lariboisière Hospital, Paris, France.

This work was funded by a Network of European Funding for Neuroscience Research grant (01EW1207) under the Seventh Framework Programme and the European Research Area Net, with the support of the French-Cerebral AutosomalDominant Arteriopathy with Subcortical Infarcts and Leukoencephalopathy Association, the PLANIOL Foundation, the NRJ Foundation, and the Leducq Foundation. The sponsor was Assistance Publique, Hôpitaux de Paris (Département de la Recherche Clinique et du Développement). unknown. Simple and robust MR imaging markers at these early stages would be of interest because future therapeutic trials will likely include the patients with the least severe diseases.

We recently showed that reaction time (RT) is a marker of subtle cognitive and behavioral alterations observed in nondisabled patients without dementia with CADASIL. ${ }^{4}$ The MR imaging correlates of RT in these patients are undetermined.

Both in healthy aging and in sporadic SVD, the corpus callosum area $\left(\mathrm{CC}_{\mathrm{A}}\right)$ reflects white matter integrity and is related to various estimates of processing speed. ${ }^{5,6}$ In the present study, we

Please address correspondence to Eric Jouvent, MD, Service de Neurologie, Hôpital Lariboisière, Two rue Ambroise Paré, 75010 Paris, France; e-mail: eric.jouvent@aphp.fr

- Indicates open access to non-subscribers at www.ajnr.org

三 Indicates article with supplemental on-line appendix.

Indicates article with supplemental on-line photos.

http://dx.doi.org/10.3174/ajnr.A5378 
aimed to test whether RT is independently related to $\mathrm{CC}_{\mathrm{A}}$ in nondisabled patients with CADASIL without dementia.

\section{MATERIALS AND METHODS Study Participants}

Twenty-six patients with genetically confirmed CADASIL followed within a prospective cohort study in the French Referral Centre for Rare Vascular Diseases of the Eye and the Brain (CERVCO, http://www.cervco.fr) were included. Within our referral center, about 250 patients with CADASIL are evaluated every 18 months with a comprehensive neuropsychological battery by an experienced neuropsychologist (S.R. or A.J.). Patients were invited to participate in the present study if they presented at the most recent evaluation (within 18 months) without global cognitive alterations as confirmed by the patient and his or her relatives; if neuropsychological testing scores were within the expected range according to the patient's age and level of education with a MiniMental State Examination score superior to 24 and did not have significant disability (modified Rankin Scale score of 0 or 1). Patients with overt motor deficits were excluded, but most reported minor poststroke residual symptoms such as gait disturbances or focal sensory deficits. Given the fluctuations of subjective cognitive and behavioral symptoms, particularly regarding fatigue and concentration issues, they are not systematically recorded at each visit and the evaluations reported in the present study are only based on objective measures. The present group of 26 patients included 22 patients for whom relationships between RT and cognitive alterations were reported previously ${ }^{4}$ and 4 additional patients recruited afterward. Twenty-nine age- and sex-matched control subjects were recruited from a local data base of healthy volunteers free of any history of neurologic or psychiatric disorders (except migraine). None of the patients or controls had a history of head trauma or demyelinating disease. An ethics committee validated the study protocol. All patients and controls gave their informed consent for participating in the present study.

\section{Neuropsychological Assessment}

All participants underwent a comprehensive neuropsychological evaluation, details of which have been published previously. ${ }^{4} \mathrm{RT}$ was measured with a simple computerized task. Subjects were asked to click a mouse button as soon as a white $\mathrm{X}$ appeared on the screen for a maximum duration of $2000 \mathrm{~ms}$. Twenty stimuli were presented sequentially during each trial, and 4 trials were performed in a row. During trials 2 and 3, the visual stimulus was primed by an auditory stimulus presented with a random time interval varying between 100 and 1000 ms. RT was recorded in milliseconds for each subject on the basis of the complete results obtained after 4 trials. We used the median RT based on the 4 trials for analyses. The total duration of this simple task was about 5 minutes. The Mini-Mental State Examination was used as a measure of global cognitive performance, ${ }^{7}$ and the presence of depression was defined according to the Diagnostic and Statistical Manual of Mental Disorders. 4th ed. Text Revision criteria, ${ }^{8}$ and the Montgomery-Asberg Depression Rating Scale score was obtained for all subjects. ${ }^{9}$ Subjects were considered apathetic if they had a Starkstein Apathy Scale score of $\geq 14$ and fulfilled the Starkstein Structured Clinical Interview for Apathy criteria. ${ }^{10}$

\section{MR Imaging Acquisition and Processing}

The original MR imaging protocol included 3D T1-weighted images obtained at $3 \mathrm{~T}$ with a Tim Trio scanner (Siemens, Erlangen, Germany) equipped with a 12-channel head coil, with a standard sagittal magnetization-prepared rapid acquisition of gradient echo sequence (in-plane resolution, $1 \times 1 \mathrm{~mm}^{2}$; section thickness, $1.1 \mathrm{~mm}$; TR, $2300 \mathrm{~ms}$; TE, $2.98 \mathrm{~ms}$; TI, $900 \mathrm{~ms}$; flip angle, $9^{\circ}$; bandwidth, $238 \mathrm{~Hz} /$ pixel; and acquisition time, 7 minutes $45 \mathrm{sec}-$ onds). FLAIR and T2*-weighted sequences were not part of the present MR imaging protocol, but all patients also underwent a standard MR imaging protocol on a $1.5 \mathrm{~T}$ scanner (Signa; GE Healthcare, Milwaukee, Wisconsin) within 6 months. The FLAIR sequence was used for the segmentation of white matter hyperintensities of presumed vascular origin, and the $\mathrm{T} 2{ }^{*}$ sequence, for counting the number of microbleeds $(\mathrm{MB})$, using previously validated methodologies. ${ }^{2,11}$ Brain parenchymal fraction (BPF), defined as the ratio of brain volume to the intracranial cavity volume, and the volume of lacunes were determined from the 3D-T1 sequence in all subjects. ${ }^{12}$ Masks of lacunes were obtained manually by a trained rater. Hypointense lesions with a signal identical to that of CSF, sharp delineation, and a diameter of $>2 \mathrm{~mm}$ were selected for this segmentation. Lacunes were distinguished from perivascular spaces by using STandards for ReportIng Vascular changes on nEuroimaging recommendations, including size criterion. $^{11}$

The $3 \mathrm{D}-\mathrm{T} 1$ scans were manually registered to the midsagittal plane, with rotation transforms in Multi-image Analysis GUI (Mango; Research Imaging Institute, University of Texas Health Science Center at San Antonio; http://ric.uthscsa.edu/mango). Anterior/posterior commissures and the aqueduct of Sylvius were aligned on the interhemispheric plane. Once re-aligned, scans were processed with manual drawing in Anatomist (www. brainvisa.info) to delineate the corpus callosum on noninterpolated images (Fig 1). This approach is generally considered the reference method. ${ }^{13}$ Given the strong links between head size and the area of the corpus callosum, ${ }^{14-16} \mathrm{CC}_{\mathrm{A}}$ was normalized to the intracranial cavity volume (ICV) while raising the $\mathrm{CC}_{\mathrm{A}}$ at the power $3 / 2$ to obtain a dimensionless value $\left(\mathrm{CC}_{\mathrm{A}}^{3 / 2} / \mathrm{ICV}\right)$. Intraand interrater reliability were evaluated in a sample including 10 patients and 10 controls selected randomly from the whole sample of 55 subjects. Intraclass correlation coefficients were 0.94 and 0.92 , respectively.

To further explore the relationships between $\mathrm{CC}_{\mathrm{A}}$ and lacunes, we used a template of the corpus callosum from the ICBMDTI-81 white matter atlas (http://www.loni.usc.edu/ICBM/ Downloads/Downloads_DTI-81.shtml), ${ }^{17}$ which was thereafter projected into the native space of each patient to systematically identify lacunes within the central part of the corpus callosum.

\section{Statistical Analysis}

Statistical analyses were performed with R statistical and computing software (http://www.r-project.org). Between-group comparisons were performed with $t$ tests for continuous variables with normal distributions. Wilcoxon tests were used otherwise. For categorical variables and comparisons of proportions, $\chi^{2}$ tests were used. Multiple linear regression modeling was used to test the relationship between $\mathrm{CC}_{\mathrm{A}}$ and $\mathrm{MR}$ imaging markers or be- 


\section{CADASIL}
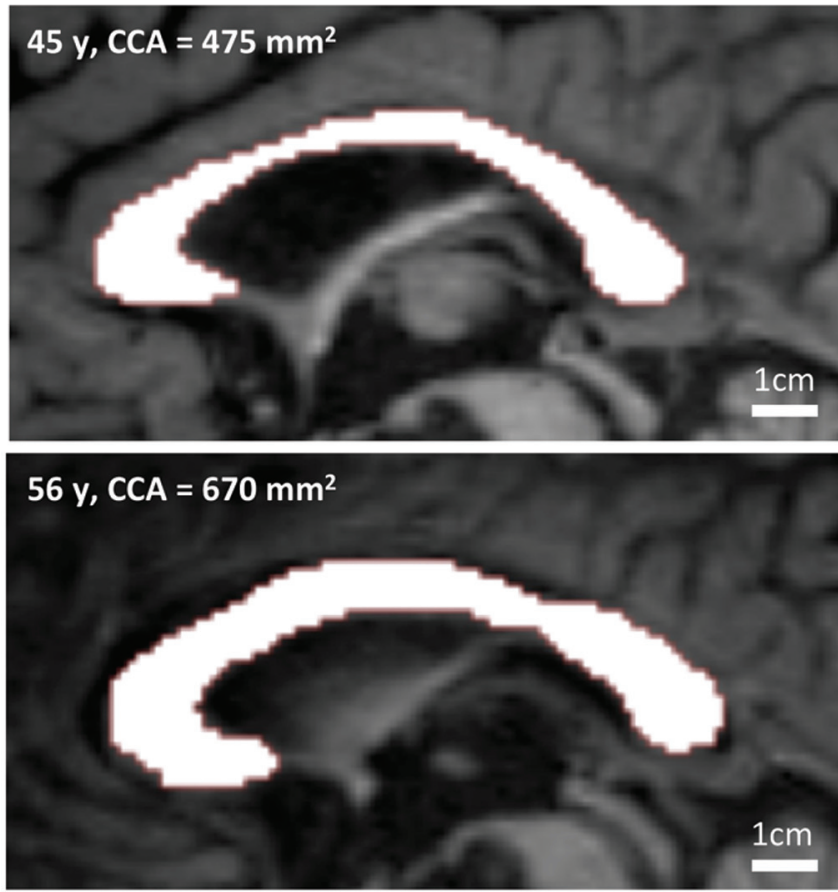

$66 \mathrm{y}, \mathrm{CCA}=581 \mathrm{~mm}^{2}$

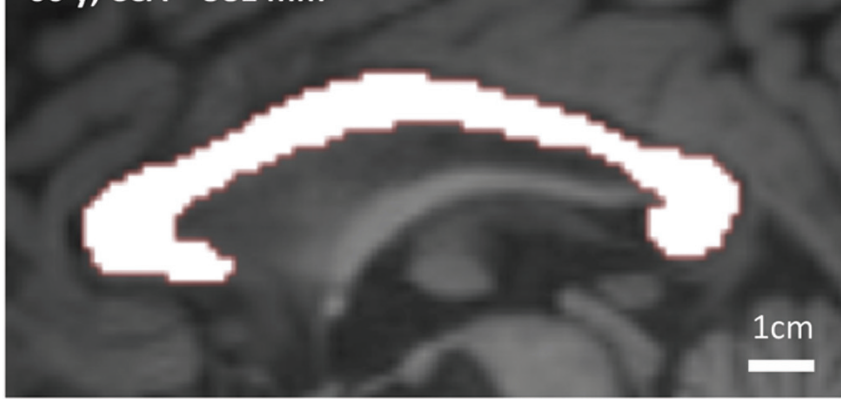

\section{CONTROLS}

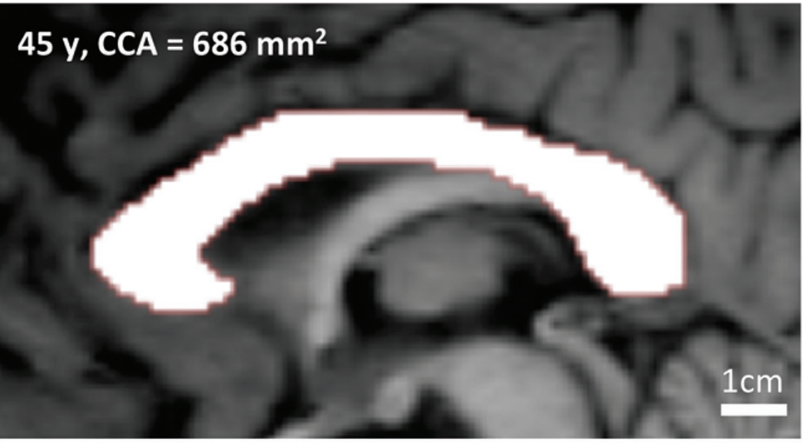

$57 \mathrm{y}, \mathrm{CCA}=720 \mathrm{~mm}^{2}$

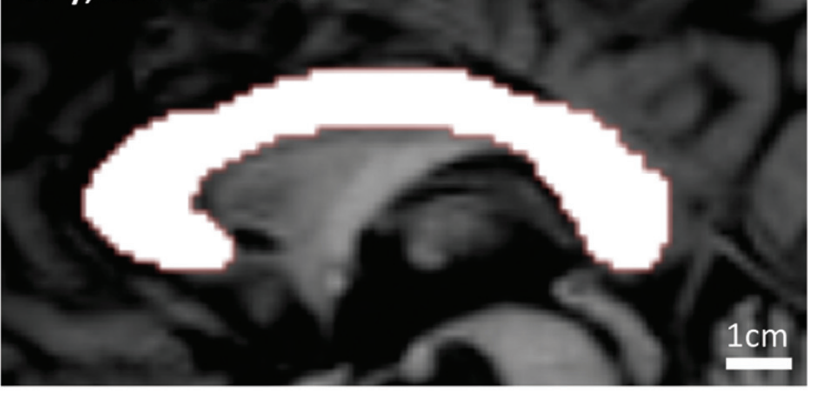

$66 \mathrm{y}, \mathrm{CCA}=779 \mathrm{~mm}^{2}$

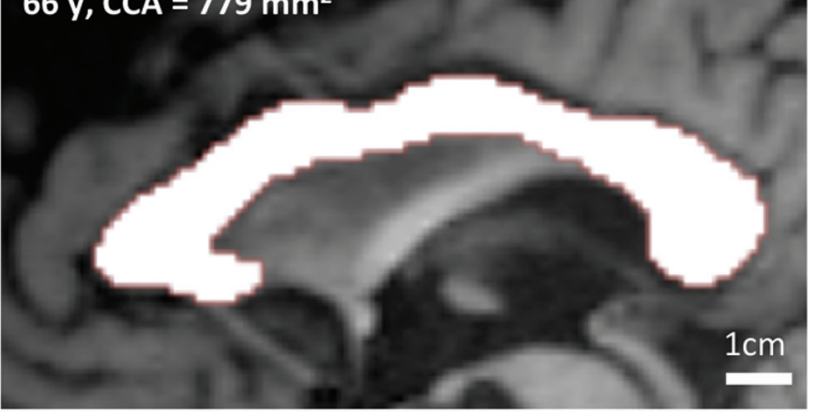

FIG 1. Examples of corpus callosum area delineation in 3 patients with CADASIL and 3 age-matched controls. Reproduction at the same scale of noninterpolated midsagittal T1-weighted images of the corpus callosum in 3 patients (left column) compared with 3 age-matched controls (right column). Corpus callosum atrophy appears obvious in some cases.

tween RT and $\mathrm{CC}_{\mathrm{A}}$. Analyses were adjusted when necessary for the following variables, based on data from the literature: age, sex, level of education, and scores of depression and apathy. For all analyses, $P<.05$ (2-tailed) was considered significant.

\section{RESULTS}

Characteristics of patients and controls are given in the Table. In line with our previous results, ${ }^{4}$ patients had significantly longer RTs than controls $(288 \pm 56 \mathrm{~ms}$ versus $253 \pm 43 \mathrm{~ms}, P=.02)$. Patients also had significantly greater scores of depression (Montgomery-Asberg Depression Rating Scale: $5.9 \pm 6.9$ versus $1.7 \pm$ $3.0, P=.007)$ and a significantly lower level of education (11.3 \pm 3.7 years versus $13.5 \pm 3.5$ years, $P=.02$ ), but the difference in RT between patients and controls remained significant after adjustment for age, sex, level of education, and scores of depression and apathy (estimate $=29.8$, standard error $[\mathrm{SE}]=14.8, P=.04$ ).

$\mathrm{CC}_{\mathrm{A}}$ was significantly smaller in patients (estimate $=1.3 \times$ $10^{-2} \pm 3.0 \times 10^{-3}$ versus $\left.1.6 \times 10^{-2} \pm 3.0 \times 10^{-3}, P<.001\right)$, even after adjustment for age and sex (estimate $=3.3 \times 10^{-3}$,
$\left.\mathrm{SE}=7.6 \times 10^{-4}, P<.001\right)$. By contrast, patients showed no brain atrophy (BPF: in patients, $0.84 \pm 0.03$, versus controls, $0.84 \pm$ $0.03 ; P=.77$ ), even after adjustment for age and sex (estimate $=$ $\left.-2.5 \times 10^{-3}, \mathrm{SE}=7.1 \times 10^{-3}, P=.72\right)$.

In patients, $\mathrm{RT}$ was negatively related to $\mathrm{CC}_{\mathrm{A}}$ both before (estimate $\left.=-7.4 \times 10^{3}, \mathrm{SE}=3.3 \times 10^{3}, P=.03\right)($ Fig 2$)$ and after adjustment for age, sex, level of education, and score of depression and apathy (estimate $=-1.2 \times 10^{4}, \mathrm{SE}=3.8 \times 10^{3}, P=.005$ ). Further adjustment for the number of $\mathrm{MB}$, the volume of white matter hyperintensities, volume of lacunes, and BPF did not alter our results (estimate $=-1.4 \times 10^{4}, \mathrm{SE}=5.3 \times 10^{3}, P=.02$ )

In patients, there was no significant association between RT and any of the classic MR imaging markers: number of $\mathrm{MB}$ (estimate $=-1.05, \mathrm{SE}=2.07, P=.62$ ), volume of white matter hyperintensities (estimate $=1.7 \times 10^{-4}, \mathrm{SE}=1.6 \times$ $10^{-4}, P=.30$ ), volume of lacunes (estimate $=-3.3 \times 10^{-2}$, $\left.\mathrm{SE}=2.5 \times 10^{-2}, P=.20\right)$ and $\mathrm{BPF}\left(\right.$ estimate $=-4.1 \times 10^{2}$, $\left.\mathrm{SE}=4.4 \times 10^{2}, P=.37\right)$. 


\begin{tabular}{|c|c|c|c|}
\hline & $\begin{array}{l}\text { Patients with } \\
\text { CADASIL }(n=26)\end{array}$ & $\begin{array}{l}\text { Control Subjects } \\
\quad(n=29)\end{array}$ & $\begin{array}{c}P \\
\text { Value }\end{array}$ \\
\hline \multicolumn{4}{|l|}{ Demographic characteristics } \\
\hline Age (mean) (yr) & $54.0 \pm 10.4$ & $53.9 \pm 11.0$ & .973 \\
\hline Male sex (No.) & $13(50.0 \%)$ & $15(51.7 \%)$ & .901 \\
\hline Right-handed (No.) & $26(100 \%)$ & $29(100 \%)$ & NA \\
\hline Level of education (mean) (yr) & $11.3 \pm 3.7$ & $13.5 \pm 3.5$ & .022 \\
\hline History of stroke (No.) (\%) & $20(77 \%)$ & $0(0 \%)$ & NA \\
\hline \multicolumn{4}{|l|}{ Neuropsychological tests } \\
\hline Reaction time (mean) (ms) & $288 \pm 56$ & $253 \pm 43$ & .02 \\
\hline MMSE score (mean) & $28.4 \pm 1.8$ & $29.0 \pm 1.1$ & .169 \\
\hline Apathy scale (mean) & $8.6 \pm 4.6$ & $6.8 \pm 3.0$ & .119 \\
\hline MADRS scale (mean) & $5.9 \pm 6.9$ & $1.7 \pm 3.0$ & .007 \\
\hline \multicolumn{4}{|l|}{ Imaging markers } \\
\hline Corpus callosum area (mean) $\left(\mathrm{mm}^{2}\right)$ & $609 \pm 93$ & $713 \pm 111$ & $<.001,<.001^{\mathrm{a}}$ \\
\hline Brain parenchymal fraction (mean) & $0.84 \pm 0.03$ & $0.84 \pm 0.03$ & .766 \\
\hline Volume of white matter (mean) $\left(\mathrm{cm}^{3}\right)$ & $450.5 \pm 66.6$ & $433.9 \pm 44.5$ & .35 \\
\hline $\begin{array}{l}\text { Volume of lacunes }{ }^{b} \text { (mean) (median) (range) } \\
\qquad\left(\mathrm{mm}^{3}\right)(n=15 / 26,57.6 \%)\end{array}$ & $529,303,14-1975$ & No control had lacunes ${ }^{c}$ & NA \\
\hline $\begin{array}{l}\text { Volume of WMH (mean) (median) } \\
\quad\left(\text { range) }\left(\mathrm{mm}^{3}\right)(n=26 / 26,100 \%)\right.\end{array}$ & 93,$638 ; 74,087 ; 7249-267,095$ & No control had significant lesions ${ }^{c}$ & NA \\
\hline $\begin{array}{l}\text { No. of } \mathrm{MB}^{\mathrm{b}} \text { (mean) (median) (range) } \\
(n=8 / 26,30.7 \%)\end{array}$ & $6.5,2.5,1-32$ & No control had $\mathrm{MB}^{\mathrm{C}}$ & NA \\
\hline
\end{tabular}

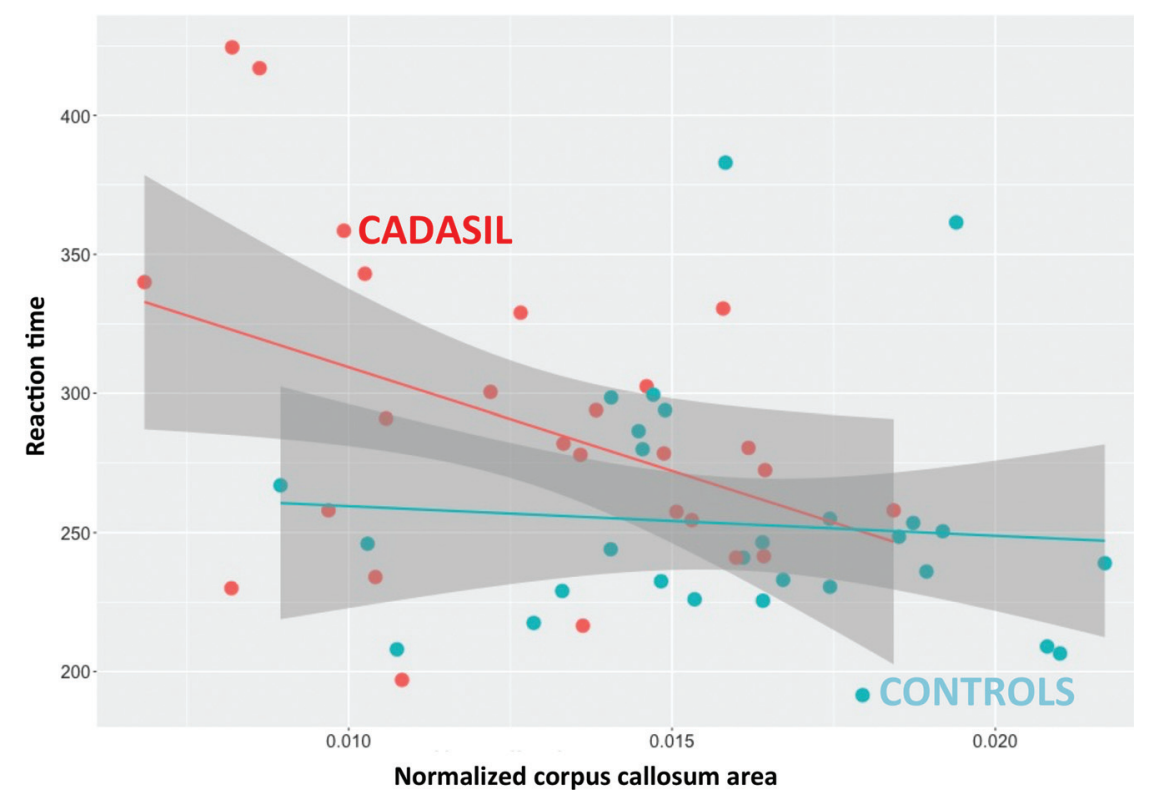

FIG 2. The relationship between reaction time and $C_{A}$ in patients with CADASIL and in controls. The mean reaction time appears significantly and negatively associated with the corpus callosum area in patients (estimate $=-7.4 \times 10^{3}, \mathrm{SE}=3.3 \times 10^{3}, P=.03$ ), while no association is observed in controls (estimate $=-1.1 \times 10^{3}, \mathrm{SE}=2.6 \times 10^{3}, P=.69$ ).

In controls, there was no significant association between RT and $\mathrm{CC}_{\mathrm{A}}$ (estimate $=-1.1 \times 10^{3}, \mathrm{SE}=2.7 \times 10^{3}, P=.69$ ).

In patients, $\mathrm{CC}_{\mathrm{A}}$ was negatively related to the volume of lacunes (estimate $=-3.1, \mathrm{SE}=0.9, P=.002$ ). Results were unchanged when we removed the 3 patients with lacunes within the central part of the corpus callosum (data not shown) from our analysis. Additionally, $\mathrm{CC}_{\mathrm{A}}$ was positively related to $\mathrm{BPF}$ (estimate $=4.6, \mathrm{SE}=1.5, P=.008)$. By contrast, there was no significant association with the volume of white matter hyperintensities (estimate $=-0.4, \mathrm{SE}=7.6, P=.96)$ or the number of $\mathrm{MB}$ (estimate $=-1.2$, $\mathrm{SE}=0.8, P=.12)$.

\section{DISCUSSION}

In the present study, we found that $\mathrm{CC}_{\mathrm{A}}$ is an independent MR imaging marker of reaction time at the early clinical stages of pure SVD. By contrast, other conventional MR imaging markers were not associated with reaction time. In this sample of patients with CADASIL without disability and without dementia who had no brain atrophy and few if any lacunes, $\mathrm{CC}_{\mathrm{A}}$ was lower than in age- and sex-matched controls. The effect of the volume of lacunes on $\mathrm{CC}_{\mathrm{A}}$ was not mediated only by the 3 patients having lacunes on its most central part. This suggests that $\mathrm{CC}_{\mathrm{A}}$ somehow summarizes the widespread effect of lacunes on its fiber bundles rather than only lacunes close to midline. Altogether, these results support the hypothesis that the accumulation of lacunes along the fibers of the corpus callosum leads to secondary degeneration translating into corpus callosum atrophy and also slows the connections between brain hemispheres, as illustrated by an increase in reaction time. While we did not observe any significant relationships between the volume of white matter hyperintensities or the number of $\mathrm{MB}$ and the corpus callosum area, smaller ischemic lesions, not visible with conventional MR imaging such as microinfarcts, might induce similar changes. 
Brain atrophy is often considered a final common pathway adding up the various consequences of vascular lesions accumulating within the brain parenchyma. ${ }^{18}$ In the present study, patients showed lower $\mathrm{CC}_{\mathrm{A}}$ but no brain atrophy compared with age- and sex-matched controls. Because of its very simple shape, detection of subtle alterations of the corpus callosum structure might be technically easier than those of the whole brain. However, given the cross-sectional nature of our study, we could not formally exclude the possibility that patients with CADASIL have innate lower $\mathrm{CC}_{\mathrm{A}}$ compared with healthy subjects, but some arguments contradict this hypothesis. First, in the present study, we found that $\mathrm{CC}_{\mathrm{A}}$ is independently associated with the volume of lacunes, suggesting that reduction of $\mathrm{CC}_{\mathrm{A}}$ might be secondary to degeneration of long-range fibers passing through the altered parenchyma, in line with the description of remote cortex atrophy after subcortical infarcts. ${ }^{19}$ Note that we observed, in 10 of 26 patients, imaging aspects compatible with such secondary changes after the occurrence of lacunes in the corpus callosum fibers (Fig 3). Also, in additional analyses, we compared 28 other patients with CADASIL without lacunes from our national cohort study who were age- and sex-matched to the control group of the present study. When considering the difference in field strengths possibly altering $\mathrm{CC}_{\mathrm{A}}$ delineation, $\mathrm{CC}_{\mathrm{A}}$ in patients with CADASIL was not significantly different from that of age- and sex-matched controls (On-line Appendix and On-line Figs 1-5). Altogether, these results strongly support that $\mathrm{CC}_{\mathrm{A}}$ is actually lower in patients as a consequence of the accumulation of vascular lesions throughout the brain parenchyma and, thus, reflects the disease severity.

Given that $\mathrm{CC}_{\mathrm{A}}$ was negatively related to the volume of lacunes and positively related to brain atrophy, it might be considered a marker of white matter atrophy. However, several reports have now shown that the presence of lacunes in the white matter is associated with widespread cortical microinfarcts that may themselves explain these relationships independent of direct white matter damage. ${ }^{20}$ While the results of the present study highlight the potential interest in $\mathrm{CC}_{\mathrm{A}}$ as a marker of SVD, the mechanisms leading to a smaller $\mathrm{CC}_{\mathrm{A}}$ in patients with CADASIL remain beyond the reach of our methodology.

The mechanisms leading to reaction time slowing remain largely unknown. While it may be tempting to look deeper into the relationships between reaction time and specific brain structure and/or networks, one must keep in mind that even in healthy subjects, the mechanisms underlying reaction time are strongly debated, with some authors considering it a stochastic process unrelated to specific brain areas or networks. ${ }^{21}$ Note that the main assumption underlying our results is that reaction time involves efficient interhemispheric cooperation. Further studies including more advanced methods such as assessment of fiber tracts, as previously performed in CADASIL, will be needed to further understand the mechanisms of reaction time slowing in this disorder. $^{22}$

Processing speed has previously been associated with $\mathrm{CC}_{\mathrm{A}}$ in sporadic forms of SVD and in other brain disorders. ${ }^{5,23-25} \mathrm{Re}-$ garding reports of sporadic forms of SVD, the absence of control subjects did not allow formally disentangling the effects of age from those of SVD in these studies. Moreover, study samples were

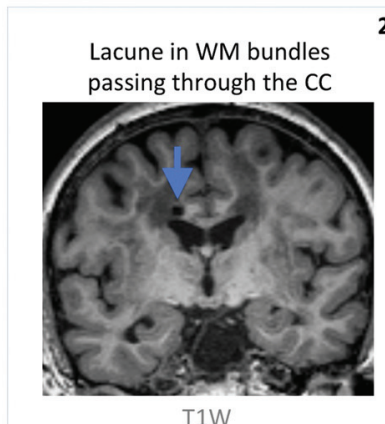

T1W

New lacune more anterior in through the $\mathrm{CC}$

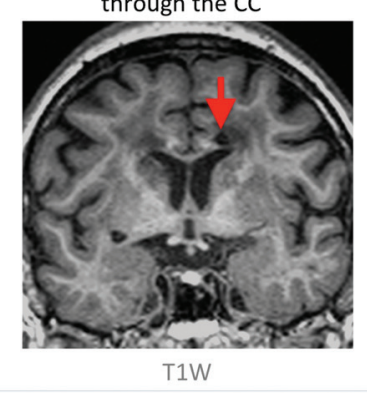
WM bundles passing
Degeneration in the $\mathrm{CC}$

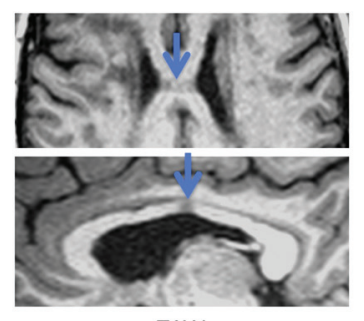

T1W

2013

Secondary degeneration in the $\mathrm{CC}$ (red) anterior to the previous one (blue)

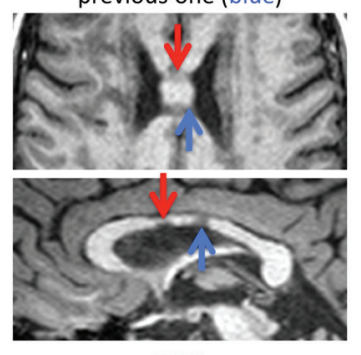

T1W
2015

CC atrophy following secondary degeneration

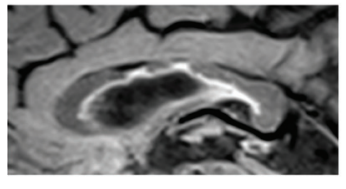

FLAIR

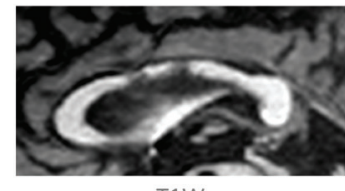

T1W
FIG 3. Corpus callosum atrophy through the appearance of lacunes and secondary white matter degeneration. An example of a 56-yearold patient with CADASIL presenting in 2010 with hypointense T7weighted signal in the corpus callosum (CC), presumably as a consequence of a lacune located in white matter bundles passing through the CC body (top panel). In 2013, a new lacune occurred in bundles passing through the $\mathrm{CC}$, localized anterior to the previous one and leading to another hypointense area in the CC (middle panel). Images acquired in 2015 show progressive CC atrophy due to degeneration secondary to the appearance of lacunes (bottom panel). TIW indicates T1-weightd.

heterogeneous, including older patients, with a significant proportion probably having associated Alzheimer disease (29\% of diagnosed dementia on a 3-year follow-up ${ }^{6}$ ). Here, the significantly larger $\mathrm{CC}_{\mathrm{A}}$ in controls, with no relationship with $\mathrm{RT}$, strongly supports our observation of a specific effect of the disease. Further studies relying, for instance, on diffusion tensor imaging may be of interest to locally quantify the effects of the disease on the corpus callosum structure.

Our study has various limitations. First, study samples were small, including only 26 patients. Given the very strict inclusion criteria of the present study, patient recruitment is challenging and prevents studying large cohorts of patients at this stage of the disease. However, despite small sample sizes, we observed significant and coherent results, emphasizing the strength of the observed relationships. The use of several covariates in multiple regression analyses with such sample sizes may lead to overfitting of the data, but the coherence of our results argues against this hypothesis. More elaborate MR imaging markers, requiring inten- 
sive postprocessing methods, might also be related to reaction time. However, we chose to restrict our analyses to the corpus callosum area to provide robust and easy-to-obtain markers for therapeutic trials. Finally, given that most patients had strokes before being prospectively followed, we were not able to determine exactly, for each patient, which of the lacunes initially led to stroke symptoms. We were thus unable to explore separately the relationships between $\mathrm{CC}_{\mathrm{A}}$ and lacunes that led to stroke and between $\mathrm{CC}_{\mathrm{A}}$ and "silent" lacunes.

Our study also has strengths. We tested a clear and unique hypothesis with a straightforward methodology, with the use of a simple MR imaging parameter, easily obtained manually and strongly correlated with volumetric measures obtained with methods requiring postprocessing skills. Our patients represent a homogeneous sample of a pure vascular disorder, including patients of various ages, allowing differentiating the effects of disease from those of age. Additionally, the detection of relationships between reaction time and corpus callosum area in small samples implies a large effect size. In patients, the effect of corpus callosum thinning was roughly twice that of aging in controls regarding an increase of reaction time.

\section{CONCLUSIONS}

We have shown that the $\mathrm{CC}_{\mathrm{A}}$ is a simple and independent imaging marker of reaction time in the early clinical stages of CADASIL. Given that clinical variability is a well-known characteristic of patients with vascular cognitive impairment, markers such as $\mathrm{CC}_{\mathrm{A}}$ can appear of high interest for future therapeutic trials in CADASIL, which will likely be performed in patients with the least severe disease. Additional studies are needed to determine whether our results are translatable in the context of sporadic SVD.

\section{ACKNOWLEDGMENTS}

We thank Chantal Ginisty, Séverine Roger, and Séverine Desmidt (Translational and Applicative Neuroimaging Research Unit, NeuroSpin) for performing the MR imaging acquisitions. We also acknowledge Jocelyne Ruffié, Véronique Joly-Testaud, and Laurence Laurier for the recruitment process of patients and controls. ${ }^{*}$ Money paid to the institution.

\section{REFERENCES}

1. Chabriat H, Joutel A, Dichgans M, et al. CADASIL. Lancet Neurol 2009;8:643-53 Medline

2. Viswanathan A, Godin O, Jouvent E, et al. Impact of MRI markers in subcortical vascular dementia: a multi-modal analysis in CADASIL. Neurobiol Aging 2010;31:1629-36 CrossRef Medline

3. Chabriat H, Hervé D, Duering M, et al. Predictors of clinical worsening in cerebral autosomal dominant arteriopathy with subcortical infarcts and leukoencephalopathy: prospective cohort study. Stroke 2016;47:4-11 CrossRef Medline

4. Jouvent E, Reyes S, De Guio F, et al. Reaction time is a marker of early cognitive and behavioral alterations in pure cerebral small vessel disease. J Alzheimers Dis 2015;47:413-19 CrossRef Medline

5. Jokinen H, Ryberg C, Kalska H, et al; LADIS group. Corpus callosum atrophy is associated with mental slowing and executive deficits in subjects with age-related white matter hyperintensities: the LADIS Study. J Neurol Neurosurg Psychiatry 2007;78:491-96 Medline
6. Frederiksen KS, Garde E, Skimminge A, et al. Corpus callosum tissue loss and development of motor and global cognitive impairment: the LADIS study. Dement Geriatr Cogn Disord 2011;32:279-86 CrossRef Medline

7. Folstein MF, Folstein SE, McHugh PR. "Mini-mental state": a practical method for grading the cognitive state of patients for the clinician. J Psychiatric Res 1975;12:189-98 CrossRef Medline

8. American Psychiatric Association. Diagnostic and Statistical Manual of Mental Disorders. 4th ed. Text Revision. Arlington: American Psychiatric Association; 2000

9. Montgomery SA, Asberg M. A new depression scale designed to be sensitive to change. Br J Psychiatry 1979;134:382-89 CrossRef Medline

10. Starkstein SE, Ingram L, Garau ML, et al. On the overlap between apathy and depression in dementia. J Neurol Neurosurg Psychiatry 2005;76:1070-74 CrossRef Medline

11. Wardlaw JM, Smith EE, Biessels GJ, et al; STandards for ReportIng Vascular changes on nEuroimaging (STRIVE v1). Neuroimaging standards for research into small vessel disease and its contribution to ageing and neurodegeneration. Lancet Neurol 2013;12:822-38 CrossRef Medline

12. Peters N, Holtmannspotter M, Opherk C, et al. Brain volume changes in CADASIL: a serial MRI study in pure subcortical ischemic vascular disease. Neurology 2006;66:1517-22 CrossRef Medline

13. Herron TJ, Kang X, Woods DL. Automated measurement of the human corpus callosum using MRI. Front Neuroinform 2012;6:25 CrossRef Medline

14. Häncke L, Staiger JF, Schlaug G, et al. The relationship between corpus callosum size and forebrain volume. Cereb Cortex 1997;7:48-56 CrossRef Medline

15. Bishop KM, Wahlsten D. Sex differences in the human corpus callosum: myth or reality? Neurosci Biobehav Rev 1997;21:581-601 CrossRef Medline

16. Sullivan EV, Rosenbloom MJ, Desmond JE, et al. Sex differences in corpus callosum size: relationship to age and intracranial size. $\mathrm{Neu}$ robiol Aging 2001;22:603-11 CrossRef Medline

17. Mori S, Oishi K, Jiang H, et al. Stereotaxic white matter atlas based on diffusion tensor imaging in an ICBM template. Neuroimage 2008;40:570-82 CrossRef Medline

18. O'Sullivan M, Jouvent E, Saemann PG, et al. Measurement of brain atrophy in subcortical vascular disease: a comparison of different approaches and the impact of ischaemic lesions. Neuroimage 2008; 43:312-20 CrossRef Medline

19. Duering M, Righart R, Wollenweber FA, et al. Acute infarcts cause focal thinning in remote cortex via degeneration of connecting fiber tracts. Neurology 2015;84:1685-92 CrossRef Medline

20. van Veluw SJ, Hilal S, Kuijf HJ, et al. Cortical microinfarcts on 3T MRI: clinical correlates in memory-clinic patients. Alzheimers Dement 2015;11:1500-09 CrossRef Medline

21. Siettos CI, Smyrnis N. Reaction time as a stochastic process implemented by functional brain networks. Cogn Neurosci 2017;8:133-35 CrossRef Medline

22. Mascalchi M, Pantoni L, Giannelli M, et al. Diffusion tensor imaging to map brain microstructural changes in CADASIL. J Neuroimaging 2017;27:85-91 CrossRef Medline

23. Jokinen H, Lipsanen J, Schmidt R, et al; LADIS Study Group. Brain atrophy accelerates cognitive decline in cerebral small vessel disease: the LADIS study. Neurology 2012;78:1785-92 CrossRef Medline

24. Karbasforoushan H, Duffy B, Blackford JU, et al. Processing speed impairment in schizophrenia is mediated by white matter integrity. Psychol Med 2015;45:109-20 CrossRef Medline

25. Bergendal G, Martola J, Stawiarz L, et al. Callosal atrophy in multiple sclerosis is related to cognitive speed. Acta Neurol Scand 2013;127: 281-89 CrossRef Medline 